\title{
Mesophilic Anaerobic Digestion of JUNCAO for Biogas Production: Structure and Functional Analysis of Microbial Communities Based on High- Throughput Sequencing
}

\section{Yuyang Qiu}

Fujian Agriculture and Forestry University College of Life Science

Yating Lei

Fujian Agriculture and Forestry University College of Life Science

Hui Zhao

Fujian Agriculture and Forestry University College of Life Science

Xiaoyu He

Fujian Agriculture and Forestry University College of Life Science

\section{Ying Huang}

Fujian Agriculture and Forestry University

Bin Liu (D liubin618@hotmail.com)

Fujian Agriculture and Forestry University

\section{Research Article}

Keywords: Arundo donax cv. Lvzhou No.1, Pennisetum giganteum z.x.lin, Mesophilic anaerobic digestion, Biogas production, Microbial community structure

Posted Date: September 24th, 2021

DOl: https://doi.org/10.21203/rs.3.rs-921298/v1

License: (c) (1) This work is licensed under a Creative Commons Attribution 4.0 International License. Read Full License 


\section{Abstract}

JUNCAO, as energy grass, was used in mesophilic anaerobic digestion (MAD) to produce methane, which has a huge market potential in biomass energy. This study was to investigate the characteristics of MAD of Arundo donax cv. Lvzhou No.1 (Lvzhou No.1) and Pennisetum giganteum z.x.lin (P. giganteum) (the growth cycle of 5 months), explore the relationship between microbial community structure and its function during MAD process. The results showed that the cumulative biogas production of Lvzhou No.1 and $P$. giganteum reached up to $370.37 \mathrm{~mL} / \mathrm{g}$ VS and $313.04 \mathrm{~mL} / \mathrm{g} \mathrm{VS}$, respectively. And the maximum methane concentration of both reached $75 \%$. The volatile fatty acid (VFA) showed a trend of increasing at first then decreasing. Microbiota analysis based on high-throughput sequencing technology showed that the same microflora could differentiate into different microflora due to different fermentation materials. Firmicutes, Bacteroidetes and Proteobacteria were the dominant bacterial phyla with two predominant genera of Unidentified_Clostridiales and Romboutsia. In addition, Euryarchaeota and Methanosaeta were the dominant archaeal phyla and genus, respectively. Spearman correlation analysis showed that the production of acidic substances in this system was mainly the reaction effect of bacteria, and the methanogenic function was mainly associated with the dominant flora of archaea. In conclusion, this study would provide new evidence for MAD of JUNCAO as new energy resources, which would pave the way for large-scale MAD of energy plants in the future.

\section{Highlights}

- It is first time to study the potential of mesophilic anaerobic digestion of JUNCAO.

- Biogas production capacity was achieved at $370.37 \mathrm{~mL} / \mathrm{g}$ VS and $313.04 \mathrm{~mL} / \mathrm{g}$ VS of Arundo donax cv. Lvzhou No.1 and Pennisetum giganteum z.x.lin, respectively.

- Production of acidic substances was mainly the reaction effect of bacteria.

- Methanogenic function was mainly associated with the dominant flora of archaea.

\section{Introduction}

Energy is a necessity for economic development and social life. However, the shortage of traditional energy and the ecological environment pollution caused by it, the increasing price of oil and the shortage of power supply are all important reasons that restrict the development of various countries [1]. Therefore, the development and application of renewable new energy is extremely urgent [2]. As an environmentfriendly clean energy, biogas can be widely used in social life and can effectively alleviate the problem of energy shortage. The raw materials for biogas production are abundant, which can be derived from various organic matters, they were degraded by a variety of microorganisms to produce a mixture of combustible gas under anaerobic conditions, that is biogas [3]. The main component of biogas is methane, which can be decomposed into $\mathrm{C}$ and $\mathrm{H}$. Therefore, biogas is also called a natural and pollution-free biomass energy, and plays a pivotal role in global economic development [4-5]. 
Traditional raw materials for biogas production are widely available, such as straw, fallen leaves, animal manure, and living garbage, but their limit output cannot enough for the large-scale production of biogas. Crop straw has low nutrient content and low methane rate, which will increase economic costs. In recent years, kinds of energy crops were found to be a good choice to replace the traditional raw materials [6-8]. As perennial energy crops are simple to plant and can be harvested for many years. They have the characteristics of high biomass yield, rapid growth and high nutrient content, which can greatly reduce the economic cost. In northern China, the extreme cold temperature can be as low as $-30{ }^{\circ} \mathrm{C}$ in winter, and in southern China, the continuous high temperature can reach $40{ }^{\circ} \mathrm{C}$ in summer. Therefore, it is necessary to develop energy crops with cold resistance, high temperature resistance and drought resistance, which is of great significance to biogas fermentation industry of China.

JUNCAO is one kind of energy crops, such as Arundo donax cv. Lvzhou No.1 (Lvzhou No.1) and Pennisetum giganteum z.x.lin (P. giganteum). Lvzhou No.1 is a kind of perennial plant belong to Gramineae with thick and erect straw, which has rapid growth rate with well-developed root system. More importantly, Lvzhou No.1 can grow normally at $-20^{\circ} \mathrm{C}$, which solves the problem of overwintering energy grass planting in the plateau and the cold northern region [9-10]. P. giganteum, a perennial erect tufted plant of the Gramineae, usually grows in tropical and subtropical regions, which has the characteristics of high yield and excellent adversity acclimation [11-12]. Both Lvzhou No.1 and P. giganteum have been regarded as good new material sources for the industrial production of biogas, but they have not been fully studied at present.

Anaerobic digestion could be carried out at a certain temperature range (psychrophilic, mesophilic, thermophilic or hyperthermophilic), but most anaerobic digesters are reacted in the mesophilic and thermophilic range because of a balance between energy and efficiency [13-14]. Therefore, compared to thermophilic anaerobic digestion (TAD), mesophilic anaerobic digestion (MAD) has the advantages of high stability and low disposal costs [15-16]. MAD is more economic and feasible, can be widely used in industry [17].

Some materials of JUNCAO were mainly degraded by fermentation microflora of methanogenic system in MAD to produce volatile fatty acid (VFA), methane, hydrogen sulfide, methane and others. At present, a large number of studies have discovered the important role of microorganisms in anaerobic digestion through high-throughput sequencing technology. Zhang et al. [16] and Chen et al. [18] found that the diversity of bacterial communities in mesophilic digesters was greater than that in thermophilic digesters, Firmicutes and Bacteroidetes represent the two most dominant phyla within the bacterial community. While Wang et al. [19] analyzed the most abundant phylum of bacterial and fungal were Bacteroidetes and Ascomycota, respectively. Zhu et al. [20] studied the recovery of biogas from sewage sludge and paper waste, the results showed that 'Attibacteria' (OP9) became the dominant bacterial phyla at the last stage, Methanosarcina and Methanothermobacter were the dominant archaeal phyla, they all occupied the main status in methanogenic activity. None of the above studies explained the relationship between microbial community structure and function was not studied. In order to further understand the network 
relationship between anaerobic fermentation microflora and methanogenesis, it is necessary to conduct in-depth analysis both of them.

In the biogas production of biomass energy, in order to reduce the cost of planting raw materials and energy consumption of biogas production, to make up for the short plate of biogas production from energy plants in harsh environment, it is of importance to study the MAD system of superior energy plants. Therefore, the purpose of this study was to develop Lvzhou No. 1 and P. giganteum as the raw materials, and to explore the changes of the microbial community during the MAD process, as well as the relationship between community structure and its function. Our results could better discover the biogas production process of JUNCAO in MAD reactor, and lay the foundation for the large-scale MAD research of new energy plants in the future.

\section{Materials And Methods}

\subsection{Mesophilic anaerobic digestion}

Lvzhou No. 1 (5-month growth cycle) and P. giganteum (5-month growth cycle) were obtained from the plantation base of The National Engineering Research Center of JUNCAO Technology as the raw materials of MAD. The original culture which was obtained from Nanping No. 1 Ranch was inoculated into Lvzhou No. 1 in a 10-L biogas digester, and then enriched for three successive generation acclimation to obtain the starter culture. For MAD, the starter culture was inoculated in "Lvzhou No. 1" (or $P$. giganteum) and was cultured in nine 10 - L biogas digesters for $30 \mathrm{~d}$ at thermostatic incubator at $37^{\circ} \mathrm{C}$.

\subsection{Sample collection}

During the MAD process, $10 \mathrm{~mL}$ of the mixture of biogas slurry and biogas residue was collected every day, and centrifuged (GL-12B, Shanghai Anting Scientific Instrument Factory, Shanghai, China) at 5000 rpm for $5 \mathrm{~min}$. Then the resulting supernatant was collected and stored at $-20{ }^{\circ} \mathrm{C}$ for further analysis. In addition, starter culture (SC), the mixture of biogas slurry and biogas residue on day 1, 5 and 10 were aseptically collected, then the samples were centrifuged at $12,000 \mathrm{rpm}$ for $10 \mathrm{~min}$ at $4{ }^{\circ} \mathrm{C}$, and the pellets were stored at $-80^{\circ} \mathrm{C}$ before further analysis. The samples collected of Lvzhou No. 1 (or P. giganteum) on day 1, 5 and 10 were labeled as LD1 (PD1), LD5 (PD5) and LD10 (PD10).

\subsection{Determination of Biogas Production, Methane Concentration}

The biogas yields were measured by vacuum method of drainage. The methane concentration in biogas was determined by a biogas analysis meter (BX568, Henan Hanwei Electronics Co., Ltd.).

\subsection{Determination of VFA Content and pH}

The $\mathrm{pH}$ was measured by $\mathrm{pH}$ meter (Mettler Toledo, FiveEasy Plus, Switzerland). The method for the determination of VFA content in biogas slurry is referred to in the Principles and Applications of 


\subsection{Microbial total DNA Extraction}

Total community DNA was extracted from the pellets using the CTAB method [22]. The concentration and purity of extracted DNA were measured by a NanoDrop 2000 UV-vis spectrophotometer (Thermo Fisher, Wilmington, MA, USA), then they were checked by $2 \%$ agarose gel electrophoresis and diluted to $1 \mathrm{ng} / \mu \mathrm{L}$ with sterile water. The extracted DNA was stored at $-80^{\circ} \mathrm{C}$ for further analysis.

\subsection{Illumina sequencing of microbial communities}

The primers 515F (5'-GTG CCA GCM GCC GCG GTA A-3') and 806R (5'-GGA CTA CHV GGG TWT CTA AT$\left.3^{\prime}\right)$ were used to amplify the V4 region of bacterial 16S rRNA genes. The V4 regions of archaeal 16S rRNA gene were amplified with primers 5'-CAG YMG CCR CGG KAA HAC C-3' and 5'-GGA CTA CNS GGG TMT CTA AT-3'. All the libraries were constructed by lon Plus Fragment Library Kit 48 rxns library (Thermo Fisher Scientific company), and were qualified by Qubit quantification. And then the sequencing was conducted by the lon S5TM platform (Bejing Novogene Technology Co., Ltd. Beijing).

\subsection{Bioinformatic analysis}

Final clean reads were processed from raw reads by Cutadapt (V1.9.1) [23], and were clustered by Uparse software (Uparse v7.0.1001) to obtain OTUs (Operational Taxonomic Units) [24]. Then, microbial annotation of OTUs sequences was carried out by using Mothur method and the SSSurrNA database [25] of SILVA132 [26]. In addition, QIIME software was used to complete the integration of species information classification and generate a complete OTUs table. R software (Version 2.15.3) was used to draw the dilution curve. The Spearman correlation value of microbiota and environmental factors were calculated in R software with the corr. test function of Psych package, then the PheatMap package was used for visualization [27].

\subsection{Statistical analysis}

Statistical analysis was applied to investigate correlation by GraphPad Prism 8 software. All data were displayed as the mean \pm standard deviation (SD).

\section{Results}

\subsection{Changes in Various Components During Mesophilic Anaerobic Digestion}

With the 30-day anaerobic digestion process, the biogas production stage of Lvzhou No. 1 and $P$. giganteum concentrated at the first 10 and 15 days, followed by a significant decline of daily biogas production, respectively (Fig. 1a). What's more, the cumulative biogas production of Lvzhou No. 1 and $P$. giganteum showed steeper increasing trends with final production amount of $370.37 \mathrm{ml} / \mathrm{g} \mathrm{VS}$ and 313.04 $\mathrm{mL} / \mathrm{g}$ VS at Day 30, respectively (Fig. 1b). On the first day of fermentation, the methane concentration of 
Lvzhou No. 1 and $P$. giganteum were only about $40 \%$ and $26 \%$, respectively. As the fermentation time progressed, the methane concentration of both gradually increased to 70\% (10-day) and 75\% (30-day) and remained nearly constant at the later stage of fermentation (Fig. 1c). As for pH, the value of grasses decreased at first and then gradually increased in the first 5 days. Since then, their $\mathrm{pH}$ values have fluctuated slightly, but the overall range remains between 7 and 7.5. Within the first 10 days of fermentation, the VFA content of Lvzhou No. 1 and P. giganteums showed a trend of roughly increasing firstly and then decreasing, and reached the production peak on the first day $(1372.40 \mathrm{mg} / \mathrm{L})$ and second day (1061.18 mg/L), respectively (Fig. 1e).

\subsection{Diversity of the Microbial Communities}

As shown in Table 1, raw reads of the bacteria and archaea were 76,000 to 87,000 and 82,000 to 97,000 . Goods coverage of all bacteria and archaea were 0.998 and 0.999 , respectively. According to the combination of Shannon and Simpson indexes and other indexes (Tables 2 and 3), the species richness and evenness of bacteria in two grasses were higher than that of archaea. Moreover, the dilution curve gradually flattened when the sequencing amount was over 20000, indicating that the sequencing amount was reasonable (Fig. 2).

\subsection{Structure of the Microbial Communities}

As shown in Fig. 3a, under mesophilic condition, the bacterial communities of two grasses are composed mainly by the phyla Firmicutes, Bacteroidetes, Proteobacteria and Cloacimonetes. Firmicutes was the dominant (38.39\%) phylum in SC, and its relative abundance increased remarkably in LD1 (48.45\%) and PD1 (51.40\%), but decreased apparently in the later stage (LD10 29.29\% and PD10 35.91\%).

Bacteroidetes was another dominant phylum, whose relative abundance increased from $10.43 \%$ (SC) to $37.57 \%$ (LD5) and $35.03 \%$ (PD5) with the progress of fermentation, then their relative abundance gradually decreased in the later. Proteobacteria are abundant (19.73\%) in SC sample, but relatively low (LD10 4.0\% and PD10 4.9\%) in the later stage. The relative abundance of Cloacimonetes remained relatively low (0.39\%-6.05\%) throughout the fermentation process. At the genus level (Fig. 3b), Unidentified_Clostridiales predominated (11.5\%) in SC sample, which increased firstly (LD5 $19.2 \%$ and PD5 15.9\%) and then declined gradually (LD10 3.2\% and PD10 5.5\%) within the fermentation process. The abundance of Romboutsia was highest $11.2 \%$ in SC, but decreased to $4.4 \%$ (LD10) and $4.3 \%$ (PD10). Ruminofilibacter and Sedimentibacter were found to have a same trend, their relative abundance was low in SC sample, but gradually increased after entering the MAD system.

As shown in Fig. 4a, Euryarchaeota was a very important and dominant phyla of Archaea. As for Lvzhou No. 1, Euryarchaeota had a higher relative abundance (71.2\%) of SC, consecutively decreased from $43.6 \%$ (LD1) to $29.1 \%$ (LD5), and increased slowly later. As for $P$. giganteums, an increasing trend was found for the relative abundance of Euryarchaeota within 10 days, with highest amount of $40.7 \%$ (PD10), which indicated that Euryarchaeota maybe more active in the later fermentation period. At the genus level, Methanosaeta was the dominant genus, occupying for almost $69.2 \%$ in SC sample. As for Lvzhou No. 1, 
the relative abundance of Methanosaeta decreased to $27.1 \%$ (LD5) and slightly increased to $36.2 \%$ (LD10). As for P. giganteums, Methanosaeta has a gradually increase trend from 26.3-39.0\% (from Day1 to Day 10). In addition, we can also see the presence of other methanogens with low relative abundance, such as Methanosarcina, Methanomassiliicoccus, Methanobacterium, Methanoculleus, etc.

\subsection{Correlation analysis between microbes and environmental factors}

Figure 5a shows the relationship between bacterial communities and environmental factors (daily biogas production, daily methane production, $\mathrm{pH}$ and VFA). Daily biogas production (BC) had a correlation with Desulfomicrobium, and daily methane production (MC) was positively related to Desulfomicrobium and Lentimicrobium. $\mathrm{pH}$ had a positive correlation with Bacteroides, Roseimarinus and Wolinella, but was negatively correlated with Unidentified_clostridiales and Ruminofilibacter. VFA was positively correlated with Ruminofilibacter. Also, the relationship between archaeal communities and environmental factors is shown in Fig. 5b. pH had a positive relation with Proteiniphilum, Methanosarcina, Anaerocella, Longilinea, Unidentified_Christensenellaceae and Anaerolinea, but negative relation with Sphaerochaeta and Methanoculleus. VFA was found to be positively related to Sphaerochaeta, Methanoculleus and Unidentified_Rikenellaceae, while negatively correlated with Anaerolinea and Unidentified_Cloacimonetes.

\section{Discussion}

Cumulative biogas production of MAD increased steadily over time, which the daily biogas production remained at a relatively high level within 15 days (Fig. 1a), corresponding with increases up to $370.37 \mathrm{ml} / \mathrm{g}$ VS and $313.04 \mathrm{~mL} / \mathrm{g}$ VS of Lvzhou No. 1 and P. giganteum on the 30th days (Fig. 1b), respectively. Over the same period of 10 days, the biogas production $(312.7 \mathrm{~mL} / \mathrm{g}$ VS) of Lvzhou No. 1 in TAD reactor studied by Lei [28] was higher than that of our study $(296.32 \mathrm{~mL} / \mathrm{g} \mathrm{VS})$, but our biogas production was obviously higher than Lei's by the time of 30 days. What's more, we also found that the biogas production fluctuated greatly in Lei's TAD process, so we guessed that its stability was not good enough. In the case of equal biogas production, our study of MAD has the advantages of good stability and low energy consumption. In terms of comprehensive economic efficiency, our MAD system was found to be feasible. What's more, compared with previous studies on biogas production of MAD using traditional materials, the fermentation system of our study has higher biogas production [29-31]. This indicates that Lvzhou No. 1 and P. giganteum as promising energy grasses are more conducive to biogas production than traditional materials in MAD system.

MAD is actually a process in which organic materials were degraded by microorganisms to produce biogas. During the initial period of anaerobic digestion, macromolecular organic matter was consumed by active microbial communities; that is, complex organic matter such as carbohydrates, proteins and lipids were hydrolyzed into monosaccharides, amino acids and long-chain fatty acids, then these small molecules were acidified by fermentation bacteria into a mixture of VFA and other secondary products [32-33]. The VFA was relatively high on the first 10 days of fermentation, and the dynamic trend of $\mathrm{pH}$ 
value was inversely proportional to VFA. The VFA reached the peak on the second for Lvzhou No. 1 and third day for $P$. giganteum, respectively (Fig. 1e), the pH value reached its lowest value at this point (Fig. 1d). At the same time, the VFA was further converted by acetogenic bacteria into acetic acid, $\mathrm{CO}$ and $\mathrm{H}_{2}$, which provided direct substrates for the methanogens to produce $\mathrm{CH}_{4}, \mathrm{H}_{2} \mathrm{~S}, \mathrm{CO}_{2}$ and other mixed biogas [34]. This was consistent with the result of 3.1, which confirmed that methanogens were relatively active in the first ten days of fermentation, and biogas production increased when VFA was decomposed by fermentation. The nutrients in the system were gradually consumed by bacteria during the anaerobic digestion process, resulting in a gradual decrease of VFA and a stable value of $\mathrm{pH}$. In the absence of nutrients, methanogens were gradually inactivated to produce less biogas (Fig. 1a and Fig. 1b). Similar results were also reported by Huang, Ohemeng-Ntiamoah and Zhu [20, 35-36].

The bacterial groups at the phyla level for all samples of Lvzhou No. 1 and P. giganteum are referred to in Fig. 3. It was observed that Firmicutes and Bacteroidetes were predominant, followed by Proteobacteria, similar results were also previously reported [37-38]. Firmicutes and Bacteroidetes were found to be the main bacteria in anaerobic digestion [39-41]. The relative abundance of Firmicutes obviously increased from day 0 to day 1 , this was caused by the increase in Clostridia-class species, such as Unidentified_Clostridiales and Romboutsia. Moreover, Clostridia-class species belong to Firmicutes phylum, and are responsible for the degradation of various substrates (sugars and proteins) as well as the production of VFAs [42]. According to Spearman correlation, Unidentified_Clostridiales had a strong negative correlation with $\mathrm{pH}$, which also indicated that Clostridium was an important bacterium in MAD.

As Bacteroidetes, the abundances of Lentimicrobium, Bacteroides and Roseimarinus were acetogenic bacteria that degrade large carbohydrate molecules to produce acid, which likely support the growth of methanogens [43-46]. Our result suggests that Lentimicrobium, Bacteroides and Roseimarinus had a higher abundance in the early and middle period of MAD. This indicated that a higher relative abundance of Bacteroidetes might result in a large number of organic acids to reduce $\mathrm{pH}$ and a high biogas production rate, which was in strong accordance with the results observed in this study (Fig. 5).

Proteobacteria have been reported to be the most dominant group in propionate-, butyrate-, and acetateutilizing microbial communities in anaerobic digesters, and the high abundance of such group might lead to higher VFA consumption and biogas production [47]. It was observed that the relative abundance of Proteobacteria was higher in the first five days, which led to the decrease of VFA and the increase of biogas production. In addition, this also well explains the decrease of VFA and biogas production in the later fermentation period.

From Spearman correlation, Desulfomicrobium was positively correlated with BC and MC (Fig. 5a). Desulfomicrobium, which uses sulfate or sulfonyl anion as terminal electron acceptor, sustains its own growth through the energy generated by anaerobic respiration and its product is $\mathrm{H}_{2} \mathrm{~S}$. Simple organic compounds (lactic acid, pyruvate, ethanol, formic acid, and hydrogen) act as electron donors for sulfate respiration. The sulfate which uses lactic acid as an electron donor does not fully breathe resulting in the 
formation of acetate and $\mathrm{CO}_{2}$. In this study, Desulfomicrobium was found to exist in the early and middle stages of biogas production (Fig. 3b), this was the main cause of $\mathrm{H}_{2} \mathrm{~S}$ and $\mathrm{CO}_{2}$ in biogas [48].

The Euryarchaeota was the most dominant archaea phyla in MAD for all samples of Lvzhou No. 1 and $P$. giganteum (Fig. 4a). Euryarchaeota mainly includes several bacterial genera (Methanogens, Halobacterium sp., Hyperthermophiles) in ecological niches, while methanogens play an important role as the dominant archaea in the biogas fermentation system [49]. During the methanogenesis process, methanogens can use acetic acid (such as acetic acid, formic acid, $\mathrm{H}_{2} / \mathrm{CO}_{2}$, methanol) produced from the hydrogen phase to produce $\mathrm{CH}_{4}$ [50-51]. Methanosaeta was predominant with a higher relative abundance in the genus level (Fig. 4b). Methanosaeta may be the most important methanogenic microorganism in nature, and they live in waste water, swamps and wetlands [52]. Our result was consistent with the study about methanogenic archaea conducted by Gaspari M [53]. In conclusion, methanogenic archaea played a very important role in MAD reactors.

\section{Conclusion}

In this study, we discussed the MAD performance of two kinds of energy grasses (Lvzhou No. 1 and $P$. giganteum). The results showed that the biogas production of Lvzhou No. 1 and P. giganteum could reached $370.37 \mathrm{~mL} / \mathrm{g}$ VS and $313.04 \mathrm{~mL} / \mathrm{g}$ VS within 30-day fermentation, respectively. And the methane concentration of both could increase to $75 \%$. The results suggested that two energy plants have good development potential and the MAD condition is feasible, which is of great significance to the production of new biogas energy. Based on the dynamic relationship between the structure and function of biogas microbial community in MAD reactor, the production of acidic substances in this system was mainly the reaction effect of bacteria, and methanogenic function was mainly associated with the dominant flora of archaea. In a word, the results of this study revealed the dynamic changes of microbial community structure and function of biogas production in MAD reactor, which created more possibilities for the development of biogas technology.

\section{Declarations}

\section{Funding Information}

This work was financially supported by grants from Natural Science Foundation of China (31370146), Fujian Agriculture and Forestry University International Cooperation and Exchange Project (No. KXG15001A), Science and Technology Innovation Fund project of Fujian Agriculture and Forestry University (KFA18055A and KFA18056A).

\section{Conflicts of interest}

The authors declare that the research was conducted in the absence of any commercial or financial relationships that could be construed as a potential conflict of interest. 
Availability of data and material

All data generated or analyzed during this work are included in this published paper.

\section{Ethics Approval and Consent to Participate}

Not applicable.

\section{References}

1. Berndes G, Hoogwijk M, Broek R (2003) The contribution of biomass in the future global energy supply: a review of 17 studies. Biomass Bioenerg 25(1):1-28. https://doi.org/10.1016/S09619534(02)00185-X

2. Wang L (2012) Research on the basic theory of biogas industrialization and resources deployment optimization of the large and medium-sized biogas project. Jilin University

3. Xiao G (2016) Inner mongolia region cow dung biogas fermentation characteristics of biogas tank based on comprehensive utilization of solar energy research. Inner Mongolia Agricultural University

4. Ma L, Tang Z, Wang C, Sun Y (2019) Research status and future development strategy of biomass energy. Bulletin of Chinese Academy of Sciences 34(04):434-442

5. Salama E, Saha S, Kurade M, Dev S et al (2019) Recent trends in anaerobic co-digestion: Fat, oil, and grease (FOG) for enhanced biomethanation. Prog Energ Combust 70:22-42. https://doi.org/10.1016/j.pecs.2018.08.002

6. Giuliana D, Roberto P, Luca C, Fabrizio A (2018) Arundo donax L. can substitute traditional energy crops for more efficient, environmentally-friendly production of biogas: a life cycle assessment approach. Bioresource Technol 267:249. https://doi.org/10.1016/j.biortech.2018.07.053

7. Corno L, Lonati S, Riva C, Pilu R et al (2016) Giant cane (Arundo donax L.) can substitute traditional energy crops in producing energy by anaerobic digestion, reducing surface area and costs: A fullscale approach. Bioresource Technol 218:826-832. https://doi.org/10.1016/j.biortech.2016.07.050

8. Weiland P (2010) Biogas production: current state and perspectives. Appl Microbiol Biot 85(4):849860. https://doi.org/10.1007/s00253-009-2246-7

9. Gao F (2020) Study on the rapid propagation technology of a new type of herbage giant fungus "oasis No.1". Henan Institute of Science and Technology

10. Wang M, Fang J, Pu Q, Miao Y et al (2020) Study on reproductive characteristics and nutritional components of the Arundo donax cv. LvzhouNo1 Journal of Plateau Agriculture 4(04):375-379+ 385

11. Peng L, Yang Y, Hou Y, Lu G (2014) The biosafety assessment of introduced Pennisetum sp. in Fujian Province, China. Fujian Journal of Agricultural Sciences 29(11):1132-1137

12. Ma S (2019) Key techniques of Pennisetum sp. cultivation in windy and cold area of northern shaanxi. J Soil Water Conserv (12):71-73 
13. Gebreeyessus G (2018) Effect of anaerobic digestion temperature on sludge quality. Waste Biomass Valori. https://doi.org/10.1007/s12649-018-0539-8

14. Suhartini S, Heaven S, Banks C (2014) Comparison of mesophilic and thermophilic anaerobic digestion of sugar beet pulp: Performance, dewaterability and foam control. Bioresource Technol 152:202-211. https://doi.org/10.1016/j.biortech.2013.11.010

15. Appels L, Baeyens J, Degrève J, Dewil R (2008) Principles and potential of the anaerobic digestion of waste-activated sludge. Prog Energ Combust 34(6):755-781. https://doi.org/10.1016/j.pecs.2008.06.002

16. Zhang Q, Zeng L, Fu X, Pan F et al (2021) Comparison of anaerobic co-digestion of pig manure and sludge at different mixing ratios at thermophilic and mesophilic temperatures. Bioresource Technol. https://doi.org/10.1016/j.biortech.2021.125425

17. Liu H, Zhong S, Wang C, Zhao S et al (2020) Biogas production from reed straw at medium temperature. China Biogas 38(01):25-29

18. Chen S, Cheng H, Wyckoff KN, He Q (2016) Linkages of Firmicutes and Bacteroidetes populations to methanogenic process performance. J Ind Microbiol Biot 43(6):771-781. https://doi.org/10.1007/s10295-016-1760-8

19. Wang X, Xu X, Bao Z, Wang H et al (2019) High-throughput sequencing analysis of microbial diversity in the combined fermentation stages of cow dung and corn straw. Food Fermentation Industries 45(03):47-55

20. Zhu A, Qin Y, Wu J, Ye M et al (2021) Characterization of biogas production and microbial community in thermophilic anaerobic co-digestion of sewage sludge and paper waste. Bioresource Technol

21. Ren N, Wang A (2004) Principles and applications of anaerobic biotechnology. Chemical Industry Press 315-317

22. Hou S, Duan J, Liang X, Ding X et al (2005) Optimized CTAB protocol for extracting the total DNA of ramie. Acta Botanica Boreali-occidentalia Sinica 25:2193-2197

23. Martin M (2011) Cutadapt removes adapter sequences from high-throughput sequencing reads. Embnet Journal 17(1). https://doi.org/10.14806/ej.17.1.200

24. Edgar RC (2013) UPARSE: highly accurate OTU sequences from microbial amplicon reads. Nat Methods 10.10:996-998. https://doi.org/10.1038/nmeth.2604

25. Quast C, Pruesse E, Yilmaz P, Gerken J et al (2013) The silva ribosomal RNA gene database project: improved data processing and web-based tools. Nucleic Acids Res 41(D1):D590-D596

26. Wang Q, Garrity G, Tiedje J, Cole J (2007) Naive Bayesian classifier for rapid assignment of rRNA sequences into the new bacterial taxonomy. Appl Environ Microbiol 73:5261-5267. https://doi.org/10.1128/AEM.00062-07

27. Algina J, Keselman H (1999) Comparing squared multiple correlation coefficients: Examination of a confidence interval and a test significance. Psychol Methods 4(1):76-83. https://doi.org/10.1037/1082-989X.4.1.76 
28. Lei Y, Xie C, Wang X, Fang Z et al (2020) Thermophilic anaerobic digestion of Arundo donax cv. Lvzhou No. 1 for biogas production: structure and functional analysis of microbial communities. Bioenerg Res 13(1). https://doi.org/10.1007/s12155-020-10105-y

29. Li J, Pang Y, Yuan H, Liu Y et al (2018) Enhancing methane production from corn stalk by mesophilic and thermophilic anaerobic digestion. China Biogas 36(01):76-80. https://doi.org/10.3969/j.issn.1000-1166.2018.01.013

30. Guo X, Zuo J, Shi X, Wang K et al (2017) Mesophilic and thermophilic anaerobic co-digestion of food waste and straw. Environ Sci 38(07):3070-3077. https://doi.org/10.13227/j.hjkx.201612267

31. Zhang M, Gao T, Chang G, Li X et al (2010) Study on mesophylic anaerobic fermentation of different mixture of respective pig and sheep manure with wheat straw. Chinese Journal of Environmental Engineering 4(09):2131-2134

32. Guilford N, Lee H, Kanger K, Meyer T (2019) Solid-state anaerobic digestion of mixed organic waste: the synergistic effect of food waste addition on the destruction of paper and cardboard. Environ Sci Technol 53(21):12677-12687. https://doi.org/10.1021/acs.est.9b04644

33. Li Y, Park S, Zhu J (2011) Solid-state anaerobic digestion for methane production from organic waste. Renew Sust Energ Rev 15(1):821-826. https://doi.org/10.1016/j.rser.2010.07.042

34. Zheng Y, Zhao J, Xu F, Li Y (2014) Pretreatment of lignocellulosic biomass for enhanced biogas production. Prog Energ Combust 42(1):35-53. https://doi.org/10.1016/j.pecs.2014.01.001

35. Huang R, Huang Z, Ran Y, Xiong X (2021) Experimental and simulation study on the surface contact between biogas fermentation liquid and straw material based on hydraulic mixing. Energy 222(1):119992. https://doi.org/10.1016/j.energy.2021.119992

36. Ohemeng-Ntiamoah J, Datta T (2018) Evaluating analytical methods for the characterization of lipids, proteins and carbohydrates in organic substrates for anaerobic co-digestion. Bioresour Technol 247:697-704. https://doi.org/10.1016/j.biortech.2017.09.154

37. Luo G, De Francisci D, Kougias P, Laura T (2015) New steady-state microbial community compositions and process performances in biogas reactors induced by temperature disturbances. Biotechnol Biofuels 8(1):3. https://doi.org/10.1186/s13068-014-0182-y

38. Na D, Pgkb C, Scd E, Ltb E et al (2021) Evolution of the microbial community structure in biogas reactors inoculated with seeds from different origin. Sci Total Environ 773. https://doi.org/10.1016/j.scitotenv.2021.144981

39. Ye N, Lü F, Shao L, Dodon J et al (2010) Bacterial community dynamics and product distribution during $\mathrm{pH}$-adjusted fermentation of vegetable wastes. J Appl Microbiol 103(4):1055-1065. https://doi.org/10.1111/j.1365-2672.2007.03321.x

40. Qiao J, Guo R, Yuan X, Shi X et al (2013) Phylogenetic analysis of methanogenic corn stalk degrading microbial communities. Environ Sci 34(04):1531-1539

41. Roest $\mathrm{K}$, Heilig $\mathrm{H}$, Smidt $H$, De Vos $W$ (2005) Community analysis of a full-scale anaerobic bioreactor treating paper mill wastewater. Syst Appl Microbiol 28(2):175-185. https://doi.org/10.1016/j.syapm.2004.10.006 
42. Yi J, Dong B, Jin J, Dai X (2014) Effect of increasing total solids contents on anaerobic digestion of food waste under mesophilic conditions: performance and microbial characteristics analysis. PLoS ONE 9. https://doi.org/10.1371/journal.pone.0102548

43. Li H, Ba Q, Yan Z, Liu X et al (2015) Studies on microbial community of different materials and anaerobic fermentation. J Environ Sci-China 35(05):1449-1457https://doi.org/10.3969/j.issn.10006923.2015.05.023

44. Tukanghan W, Hupfauf S, Gómez-Brandón M, Insam H et al (2021) Symbiotic Bacteroides and Clostridium-rich methanogenic consortium enhanced biogas production of high-solid anaerobic digestion systems. Bioresource Technol 14:100685. https://doi.org/10.1016/j.biteb.2021.100685

45. Sun L, Toyonaga M, Ohashi A, Tourlousse D et al (2016) Lentimicrobium saccharophilum gen. nov., sp. nov., a strictly anaerobic bacterium representing a new family in the phylum Bacteroidetes, and proposal of Lentimicrobiaceae fam. nov. Int J Syst Evol Microbiol 66(7):2635-2642. https://doi.org/10.1099/ijsem.0.001103

46. Wu W, Liu Q, Chen G, Du Z (2016) Roseimarinus sediminis gen. nov., sp. nov., a facultatively anaerobic bacterium isolated from coastal sediment. Int J Syst Evol Micr 65(7):2260-2264. https://doi.org/10.1099/ijs.0.000250

47. Zhang L, Ling J, Cui W, Du L (2014) Characteristics of microbial communities in full-scale biogas digesters with straw as substrate. J Agro-Environ Sci 33(03):584-592. https://doi.org/10.11654/jaes.2014.03.027

48. Whitman W (2015) Bergey's manual of systematics of archaea and bacteria. 1-9

49. Manaia C, Nogales B, Nunes $O$ (2003) Tepidiphilus margaritifer gen. nov., sp. nov., isolated from a thermophilic aerobic digester. Int J Syst Evol Micr 53(5):1405-1410. https://doi.org/10.1099/ijs.0.02538-0

50. Dong L, Cao G, Tian Y, Wu J et al (2020) Improvement of biogas production in plug flow reactor using biogas slurry pretreated cornstalk. Bioresource Technol 9:100378. https://doi.org/10.1016/j.biteb.2019.100378

51. Demirel B, Scherer $P(2008)$ The roles of acetotrophic and hydrogenotrophic methanogens during anaerobic conversion of biomass to methane: a review. Rev Environ Sci Biotechnol 7(2):173-190. https://doi.org/10.1007/s11157-008-9131-1

52. Li X, Hou L, Liu M (2019) Archaeal community structure and diversity in intertidal sediments of the Yangtze River Estuary. J Environ Sci-China 39(04):1744-1752. https://doi.org/10.19674/j.cnki.issn1000-6923.2019.0208

53. Gaspari M, Treu L, Zhu X, M Palù et al (2021) Microbial dynamics in biogas digesters treating lipidrich substrates via genome-centric metagenomics. Sci Total Environ 146296. https://doi.org/10.1016/j.scitotenv.2021.146296

\section{Tables}


Due to technical limitations, tables are only available as a download in the Supplemental Files section.

Figures

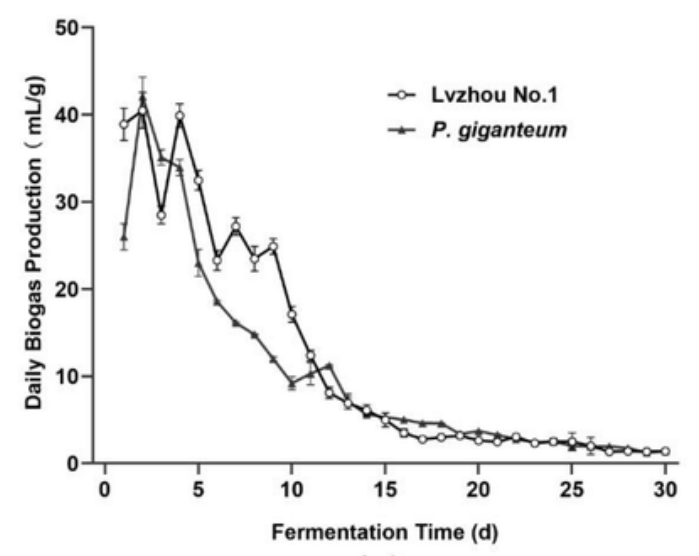

(a)

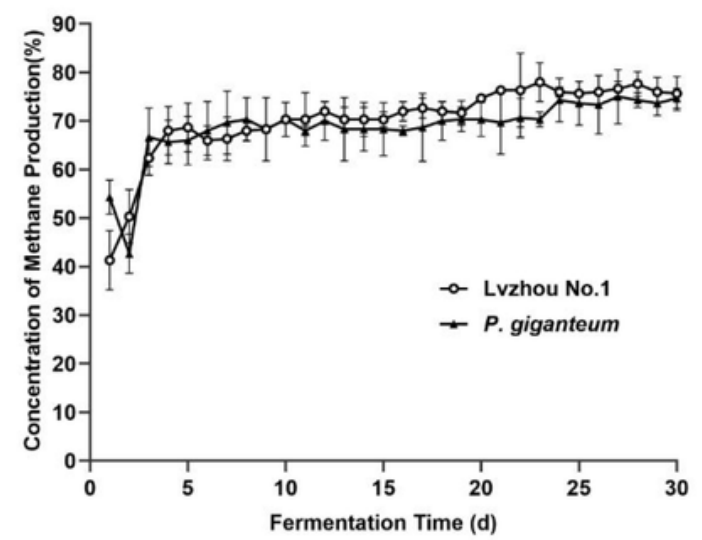

(c)

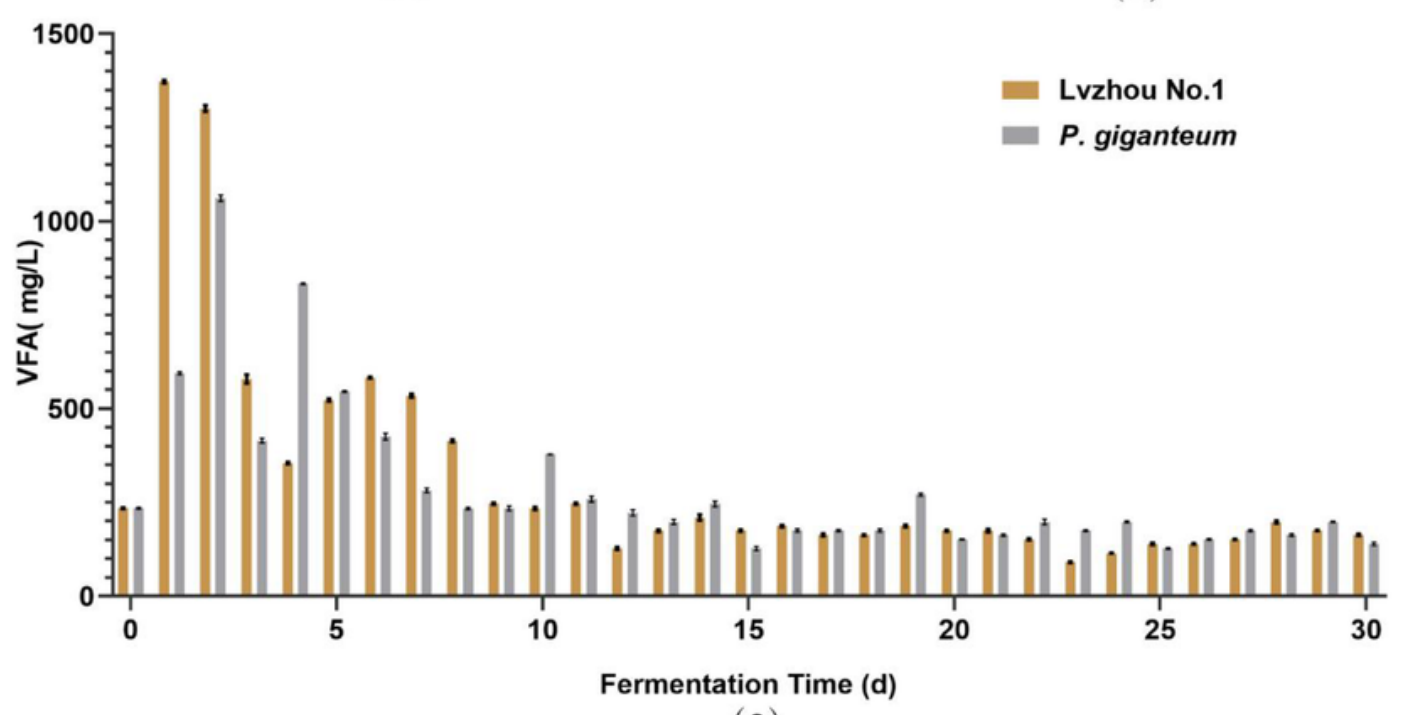

(e)

\section{Figure 1}


MAD of Lvzhou No. 1 and P. giganteum. (a) The daily biogas production amount of the medium temperature fermentation of both grass; (b) The cumulative biogas production of the medium temperature fermentation of both grass; (c) The concentration of methane produced by the medium temperature fermentation of both grass; (d) $\mathrm{pH}$ value of medium temperature fermentation biogas slurry; (e) Variation of VFA content in biogas slurry produced by medium temperature fermentation.

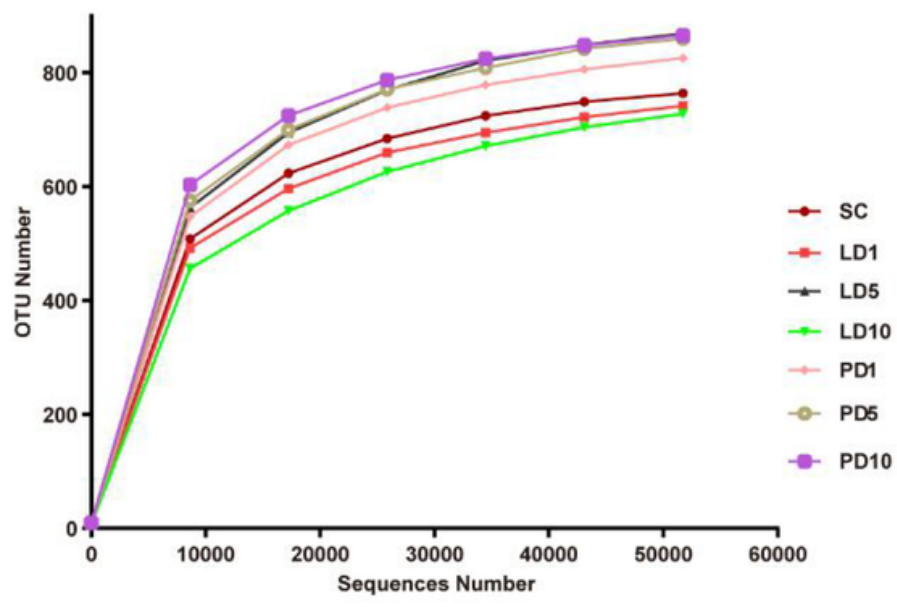

(a)

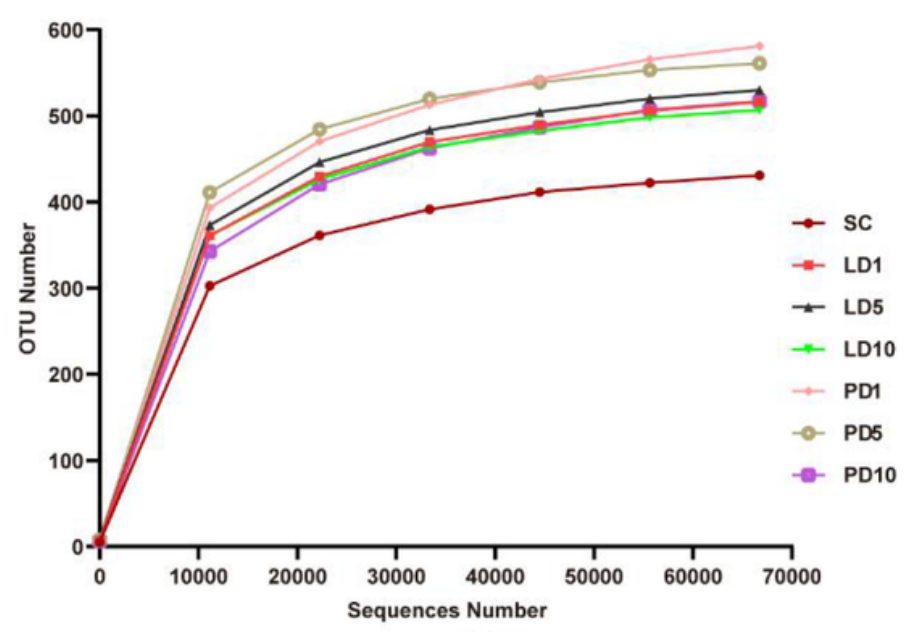

(b)

Figure 2

Rarefaction Curve of bacteria (a) and archaea (b). Notes: SC indicates bacteria of the starter culture, LD1 and PD1 indicate bacteria (or archaea) at the first day of fermentation, LD5 and PD5 indicate bacteria (or archaea) on the 5th day of fermentation, LD10 and PD10 indicate bacteria (or archaea) on the 10th day of fermentation.

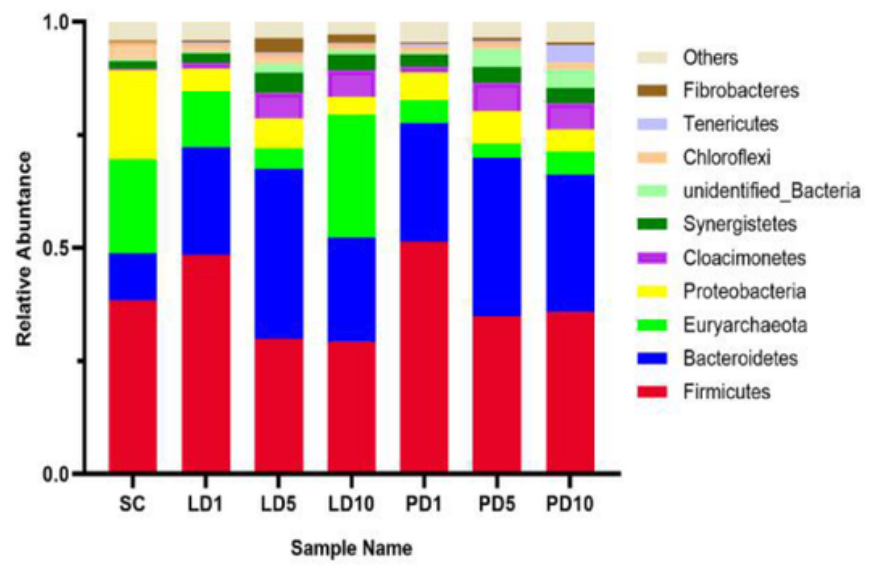

(a)

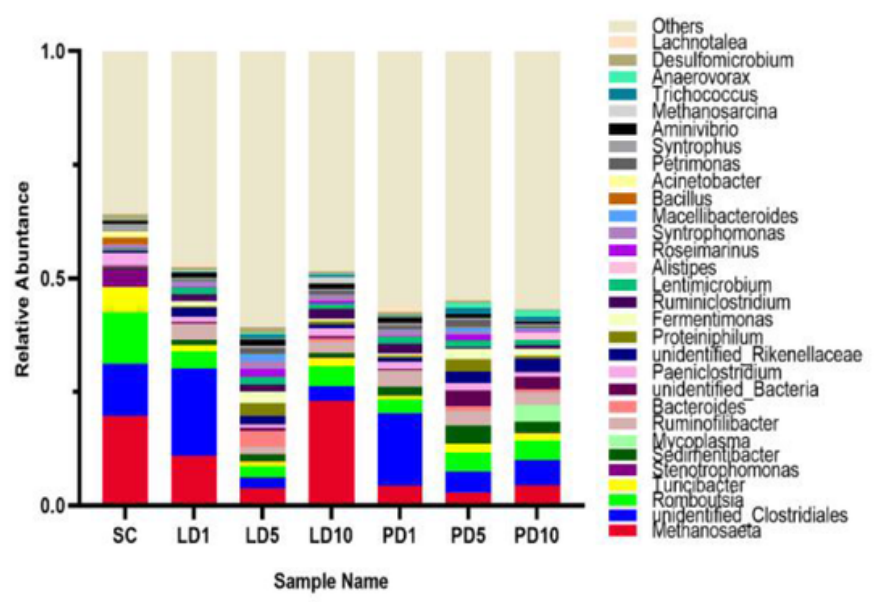

(b)

\section{Figure 3}


Histogram of the relative abundance of bacteria at the phylum and genus level. (a) Bacteria phylum. (b) Bacteria genus. Notes: SC indicates bacteria of the starter culture, LD1 and PD1 indicate bacteria at the first day of fermentation, LD5 and PD5 indicate bacteria on the 5th day of fermentation, LD10 and PD10 indicate bacteria on the 10 th day of fermentation.

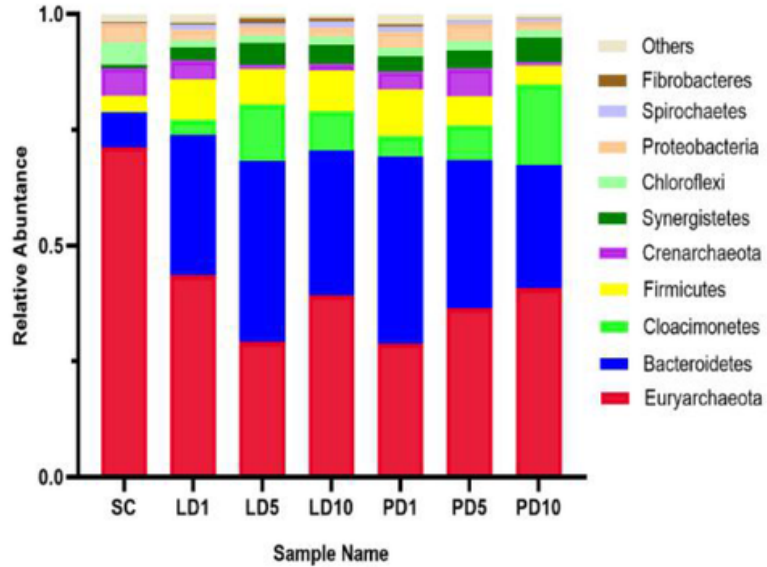

(a)



(b)

\section{Figure 4}

Histogram of the relative abundance of archaea at the phylum and genus level. (a) Archaea phylum. (b) Archaea genus. Notes: SC indicates archaea of the starter culture, LD1 and PD1 indicate archaea at the first day of fermentation, LD5 and PD5 indicate archaea on the 5th day of fermentation, LD10 and PD10 indicate archaea on the 10th day of fermentation. 


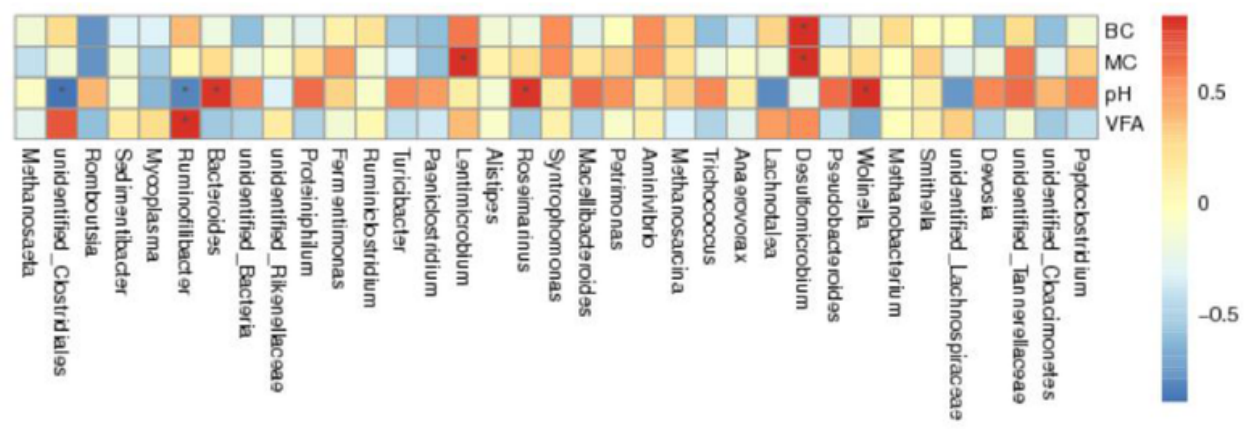

(a)

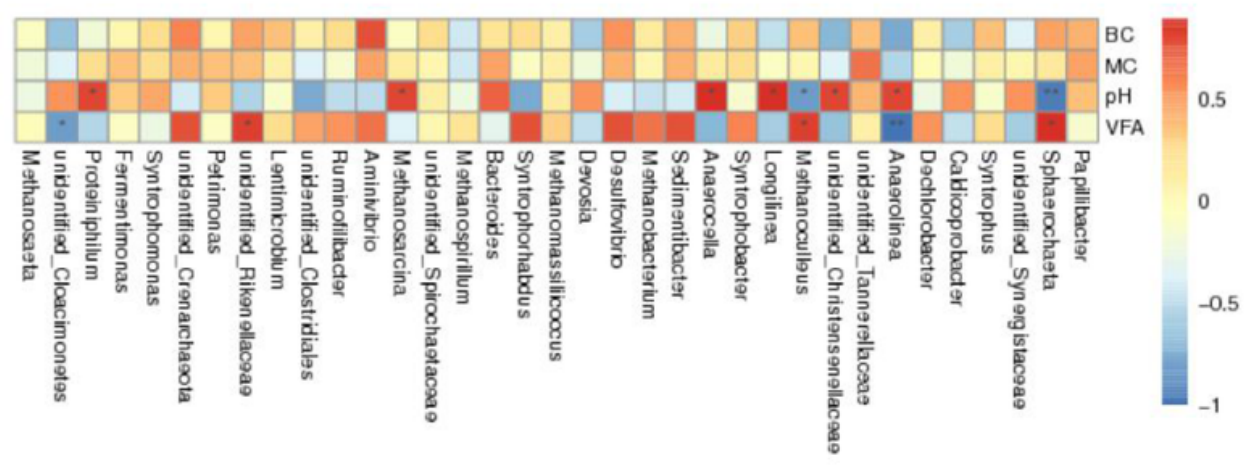

(b)

\section{Figure 5}

Heat map of Spearman correlation analysis at the genus level. (a) Bacteria, (b) Archaea. Note: BC stands for daily biogas production; MC stands for daily methane production; VFA stands for volatile fatty acid; (vertical for environmental factor information, horizontal for species information, the corresponding value in the middle heat map is the Spearman correlation coefficient $r$, which is between -1 and 1. $r<0$ is a negative correlation and $r>0$ is a positive correlation. The mark * indicates the significance test $p$ value $<0.05$.)

\section{Supplementary Files}

This is a list of supplementary files associated with this preprint. Click to download.

- GraphicalAbstract.pdf

- Table.pdf 